\title{
Special issue on simulated evolution and learning
}

\author{
Michael Kirley $\cdot$ Mengjie Zhang $\cdot$ Xiaodong Li
}

Published online: 18 November 2009

(C) Springer-Verlag 2009

\section{Introduction}

The field of natural computation is one of the fastest growing areas in computer science and engineering. Although its origins can be traced back to the cybernetics movement of the 1950s, it has undergone a renaissance since the mid-1980s, primarily due to the fundamental work by pioneers (for example, John Holland, Ken De Jong, David Goldberg, John Koza, Lawrence Fogel) in the genetic and evolutionary algorithms domain.

Problem solving techniques based on simulated evolution and learning encapsulate a wide variety of natural computation implementations. These algorithms, implemented as computer simulations, typically include a twostep iterative process: the generation of random variation followed by selection. As the model is iterated, good

The list of reviewers in alphabetical order: Hernan Aguirre, Anwar Ali Yahya Althari, Helio J.C. Barbosa, Jurgen Branke, Lam Thu Bui, Eduardo Gontijo Carrano, Siang Yew Chong, Dilip Datta, Grant Dick, Daryl Essam, Marcus Gallagher, Antony Iorio, Amitay Isaacs, Hisao Ishibuchi, Mark Johnston, Robert Ian McKay, Attila Szolnoki, Feng Yixiong, Bo Yuan, Aimin Zhou.

M. Kirley $(\bowtie)$

Department of Computer Science and Software Engineering,

The University of Melbourne, Melbourne, Australia

e-mail: mkirley@csse.unimelb.edu.au; mkirley@cs.mu.oz.au

\section{Zhang}

School of Engineering and Computer Science,

Victoria University, Wellington, New Zealand

e-mail: Mengjie.Zhang@ecs.vuw.ac.nz

X. Li

School of Computer Science and Information Technology,

RMIT University, Melbourne, Australia

e-mail: xiaodong.li@rmit.edu.au (optimal) solutions emerge. Evolutionary algorithms typically do not make assumptions about the underlying search space. As such, they provide a means for tackling hard practical problems-ones involving large, complex search spaces. Over the past decades evolutionary optimization and learning algorithms have been applied successfully to a wide variety of real-world problems.

This Special Issue brings together recent contributions in the evolutionary optimisation and learning domains, spanning a diverse range of topics. The four papers included in this Special Issue, were among the invited submissions from SEAL08 (the 7th International Conference on Simulated Evolution and Learning). Each of the submitted papers was substantially revised and extended based on the original conference version. The extended papers were then rigorously reviewed in two rounds by at least three anonymous reviewers. The high quality contributions introduced below clearly reflect the strong interest in simulated evolution and learning as a practical problem solving vehicle.

\section{The contributions}

In the paper by Kinzett, Johnston and Zhang, Numerical simplification for bloat control and analysis of building blocks in genetic programming, the issue of bloat in genetic programming is investigated. A number of techniques have been proposed to tackle the bloat problem. Here, the authors introduce a node-based numerical simplification as a tree pruning criterion to control program size. Detailed investigations of the effects of online program simplification, both algebraic and numeric, on program size and resource usage are presented. In addition, detailed analysis of the impacts of this simplification on the building blocks 
within the evolving population are presented. Significantly, the authors show that the proposed simplifications results in reductions in the expected program size and thus computation time.

The paper, Improving the performance of evolutionary algorithms in grid-based puzzles resolution, by OrtizGarcia, Salcedo-Sanz, Perez-Bellido, Carro-Calvo, Portilla-Figueras and Yao represents a significant change in direction, emphasizing the wide array of applications that may be tackled using simulated evolutionary learning approaches. The authors propose several modifications to existing hybrid evolutionary algorithms (EAs) in gridbased puzzles, using a-priori probabilities of $0 / 1$ occurrence in binary encodings. Here, two different grid based puzzles, the Japanese puzzles and the Light-up puzzle, with characteristic constraints are used as test beds for the hybrid algorithms. The authors report that the proposed initialization and operators based on a-priori probabilities of bits improvers both the outcomes and scalability properties of the evolutionary search.

Aguirre and Tanaka's paper, Adaptive epsilon-ranking on many-objective problems, introduces new techniques to improve the performance of evolutionary multi-objective optimization algorithms for many objective problems. In the proposed adaptive epsilon-ranking approach, sampled solutions keep their initial Pareto dominance rank. Solutions located within the virtually expanded epsilon-dominance regions of the sampled solutions are then demoted to an inferior rank. The epsilon parameter, which determines the expanded regions of dominance of the sampled solutions, is adapted at each generation so that the number of highest ranked solutions is kept close to a desired number that is expressed as a fraction of the population size. The authors implement their adaptive ranking using a version of NSGA-II and presented detailed numerical simulation results using a suite of benchmark problems, including the MNK-Landscape and DTLZ continuous functions, with 3 $\leq$ number of objectives $\leq 10$.

In keeping with the theme of diversity in the application domain, the paper by Rezaei and Kirley, The effects of time-varying rewards on the evolution of cooperation, investigates the evolution of cooperation in multi-agent systems based on a form of cultural learning. The authors introduce time-varying non symmetric rewards into a spatial version of the well-known Prisoner's Dilemma game. The rationale behind this approach was based on the notion that the associated payoffs from pursuing certain strategies do vary among members of real-world populations. Detailed numerical simulations show that dynamic updates to payoff values induce a change in equilibrium cooperation levels. This finding suggests that heterogeneous reward mechanisms have a direct effect on the emergent level of cooperation in the spatial Prisoners Dilemma game.

\section{Concluding comment}

We would like to thank all the people that have contributed to this Special Issue. In particular, we thank the Editor-in-Chief, Larry Bull, and Maria Mhanilet de Leon for their support and help during the process. Thank you to the numerous referees for their expertise and all the hard work allocated to the reviewing task. Finally, a special thank you to the authors who submitted their work to this 'Special Issue'. 